\title{
Clinical and Economic Impact of Glatiramer Acetate Versus Beta Interferon Therapy Among Patients With Multiple Sclerosis in a Managed Care Population
}

\author{
DANIEL A. OLLENDORF, MPH; EVGUENIA JILINSKAIA, PhD; and MERRIKAY OLEEN-BURKEY, PhD
}

\begin{abstract}
OBJECTIVE: To examine the outcomes of use of glatiramer acetate (GA) versus beta interferons-1a (intramuscular) $(1 \mathrm{~A})$ and $-1 \mathrm{~b}(1 \mathrm{~B})$ in patients with multiple sclerosis (MS) in a managed care setting.

METHODS: Data were obtained from a national retrospective claims database from January 1996 to June 2001. Patients were followed from the first prescription for immunomodulatory therapy until plan disenrollment or end of study time frame. The incidence of all relapses (defined as hospitalization for MS or ambulatory visit followed by use of systemic corticosteroids) as well as utilization and costs of MS-related care were examined for each group. Data were adjusted for variable follow-up using survival techniques.

RESULTS: A total of 8,457 patients receiving immunomodulatory therapy were included in the study cohort; follow-up averaged 17.3 months. Three quarters of patients were female; the mean age was 42.2 years. The risk of relapse (defined as number of new cases) at one year was significantly increased for the beta interferons relative to GA (hazard rates: 1.15 and 1.51 for $1 A$ and $1 B$, respectively, $P<0.01$ ). Mean $( \pm S D)$ costs of care also were reduced among GA patients $(\$ 9,522[ \pm \$ 9,706]$ versus $\$ 9,957$ $[ \pm \$ 9,083]$ and $\$ 10,185[ \pm \$ 9,526]$ for $1 \mathrm{~A}$ and $1 \mathrm{~B}$, respectively). These findings persisted in multivariate analyses, controlling for differences in demographic characteristics and propensity scores for immunomodulatory therapy.
\end{abstract}

CONCLUSIONS: Glatiramer acetate is associated with reductions in the incidence of relapse and costs of care relative to the beta interferons among this large group of managed care patients with MS.

KEYWORDS: Multiple sclerosis, Immunomodulatory therapy, Costs and costs analysis, Relapse rates

J Managed Care Pharm. 2002(8)6:469-76

\section{Authors}

DANIEL A. OLLENDORF, MPH, is Director, Analytic and Consulting Services, and EVGUENIA JILINSKAIA, PhD, is Senior Biostatistician at PharMetrics, Inc., Watertown, Massachusetts; MERRIKAY OLEEN-BURKEY, PhD, is Director, Health Outcomes Research at Teva Neuroscience, Inc., Kansas City, Missouri.

CORRESPONDING AUTHOR: Daniel A. Ollendorf, Director, Analytic and Consulting Services PharMetrics, Inc., 150 Coolidge Avenue, Watertown, MA 02472 Tel: (617) $972-$ 8590; Fax: (617) 972-8587; E-mail: dollendorf@pharmetrics.com

Copyright $\odot$ 2002, Academy of Managed Care Pharmacy. All rights reserved.
$\mathrm{M}$ ultiple sclerosis (MS) is a costly and debilitating disease. Approximately 350,000 persons in the United States are currently diagnosed with MS; it is the most common cause of chronic neurologic disability in young adults. ${ }^{1}$ While the clinical course of MS varies substantially by patient, the disease typically progresses to some level of disability in approximately two thirds of MS patients. ${ }^{2}$ Regardless of the level of disability, patients with MS often report negative psychological and social impacts from the disease. The direct medical costs of MS also are substantial. Total annual expenditures for medical services per MS patient have been reported to range from $\$ 7,000$ to $\$ 13,000$ in this country, reflecting levels 2 to 3 times higher than those for all private and public insurance enrollees. ${ }^{3}$

Historically, pharmacologic treatment of MS has revolved around amelioration of symptoms (e.g., use of benzodiazepines and muscle relaxants to control spasticity and use of corticosteroids to reduce the severity of MS relapses). Recently, however, the introduction of several new medications has changed MS management. These medications, which include glatiramer acetate (GA) (Copaxone, Teva Pharmaceutical Industries, Ltd.), a synthetic, noninterferon polypeptide of 4 amino acids, as well as the type 1 beta interferons (i.e., beta interferon-la (1A) [Avonex, Biogen Inc.] and beta interferon-1b (1B) [Betaseron, Berlex Laboratories, Inc.]) have been demonstrated to be effective in reducing the rate of relapse as well as slowing disease progression in the "relapsing-remitting" form of MS..$^{-14}$ At the time this project was completed, the newest beta interferon la (Rebif, Serono S.A.) was not commercially available in the United States.

The precise action of beta interferons in MS is unknown. However, these drugs are known to decrease lymphocyte proliferation and interferon gamma expression, induce anti-inflammatory Th2 cytokines, and most importantly to the anti-inflamatory effect, diminish the migration of activated $\mathrm{T}$ cells across the blood-brain barrier. ${ }^{15-18}$ In contrast, the observed effects of glatiramer acetate are quite distinct from those of the beta interferon and include (a) competition with myelin-basic protein (MBP) for binding to the major histocompatibility complex (MHC) molecules; (b) competition of bound glatiramer acetate and MHC with MBP/MHC for binding to the T-cell receptor; (c) induction of compound-specific Th2 cells, leading to a profound Th2 shift; and (d) migration of compoundspecific cells into the central nervous system. ${ }^{19.23} \mathrm{~A}$ fifth mechanism, that of a neuroprotective role, has also been observed in relation to optic nerve damage. ${ }^{24}$ 


\section{Clinical and Economic Impact of Glatiramer Acetate Versus Beta Interferon Therapy Among Patients With Multiple Sclerosis in a Managed Care Population}

In addition to these mechanistic differences, data from clinical trials and postmarketing surveillance studies suggest that glatiramer acetate may have several distinct advantages relative to the interferons, including a relatively mild side-effect profile allowing for less use of concomitant medications such as acetaminophen and NSAIDs, lack of neutralizing antibodies, and reduced need for laboratory monitoring. ${ }^{25}$ These advantages may be associated with greater durability of treatment, better outcomes, and reduced utilization and costs relative to interferon therapy. Patterns of pharmacotherapy as well as the costs and effects of these 3 medications have never been compared under conditions of typical clinical practice, however. To address these needs, an examination of the impact of GA on the utilization and costs of MS-related care relative to that of beta interferon therapy was undertaken, using data from a proprietary database.

\section{Methods}

\section{Overview}

Data for this study were obtained on all patients with one or more institutional or provider claims with a listed diagnosis of MS (ICD-9-CM 340) who were in the database between January 1, 1996, and June 30, 2001. Patients were then classified into 3 treatment groups based on data on the first paid pharmacy claim observed during the study period (i.e., GA, 1A, or 1B). Each patient was assigned an "index date" based on the date of the first prescription for immunomodulatory therapy.

A variety of measures were then compared during the "follow-up period" (i.e., beginning with the index date) between patients in the 3 treatment groups, including the cumulative incidence of relapses, time to first relapse, and the mean number of relapses, as well as the utilization and costs of selected MS-related medications and health care services. Because the duration of eligibility for health and drug benefits during the follow-up period differed by patient, annualization as well as techniques of survival analysis (i.e., examination of data according to time observed without any specific threshold) were employed to estimate the above-described measures.

\section{Data Source}

Data were obtained from the proprietary database, which is comprised of fully adjudicated medical and pharmaceutical claims for nearly 27 million unique patients from 43 health plans across the United States. The database includes both inpatient and outpatient diagnoses (in ICD-9-CM format) and procedures (in CPT-4 and HCPCS formats) as well as both community pharmacy and mail-order prescription records; available data on prescription records include the National Drug Code (NDC) as well as days supplied and quantity dispensed (for a subgroup of datasets). Both paid and charge amounts are available for all services rendered as well as dates of service for all claims. Additional data elements include demographic variables (age, gender, geographic region), plan type (e.g., HMO, PPO), payer type (e.g., commercial, self-pay), provider specialty, and start and stop dates for plan enrollment.

Records in the database are representative of the national managed care population on a variety of demographic measures, including geographic region, age, gender, and plan type. The data are also longitudinal, with an average member enrollment time of 2 years. Only health plans submitting data for all members are included in the database, ensuring complete data capture and unbiased samples. Data contributions are also subjected to a series of quality checks to ensure a standardized format and minimal error rates.

\section{Measures}

Measures of interest in these analyses included the incidence of all relapses as well as utilization and costs of MS-related medications and health care services during the period of follow-up. MS relapses are typically defined using one of several disability or symptom scales. ${ }^{26,27}$ Analyses of claims data are limited, however, to measures that would be associated with care-seeking behavior. An operational definition of relapses was therefore employed and was defined on the basis of either (a) an inpatient claim (hospitalization) with a listed principal diagnosis of MS or (b) a claim for an outpatient visit with a listed diagnosis of MS in combination with a pharmacy or medical claim (within 7 days after the visit) for one of the following: intravenous methylprednisolone or corticotrophin or oral methylprednisolone, prednisone, prednisolone, or dexamethasone. If multiple claims were present within a 30-day window, this was treated as a single relapse event; the first available service date within such a grouping was deemed to be the relapse date.

Medications of interest included immunomodulatory therapy (i.e., 1A [NDC code 59627-0001-03, "J" code 1825], 1B [50419-0521-03, 50419-0521-15, J1830], and GA [000881150-03, Q2010]), as well as prescription medications indicated for control of MS symptoms and side effects of immunomodulatory therapy (e.g., antispasmodics, anticholinergics, corticosteroids).

Health care services included selected laboratory tests for monitoring of immunomodulatory therapy (i.e., complete blood counts [CPT-4 code 85031, 85022-85025], platelet counts [85007, 85027, 85585, 85590, 85595], and liver function tests [80076, 82040, 82247, 82248, 84075, 84155, 84460, 84450]), outpatient services (i.e., emergency room, physician office, home health, imaging [i.e., MRI], and other hospital outpatient visits), and hospitalizations for MS. Inpatient and outpatient services were deemed to be MS-related based on a relevant listed diagnosis (principal or secondary); lab tests were tallied regardless of diagnosis. Costs of all relevant medications and services also were assessed. A health plan perspective was adopted in these analyses; cost estimates were therefore based on the amount paid (less patient copayments and deductibles) for relevant claims.

\section{Analyses}

Primary analyses were conducted on an intent-to-treat basis; all patients with an MS diagnosis and receipt of immunomodula- 
Clinical and Economic Impact of Glatiramer Acetate Versus Beta Interferon

Therapy Among Patients With Multiple Sclerosis in a Managed Care Population

TABLE 1 Demographic and Clinical Characteristics of the Study Sample by Treatment Group

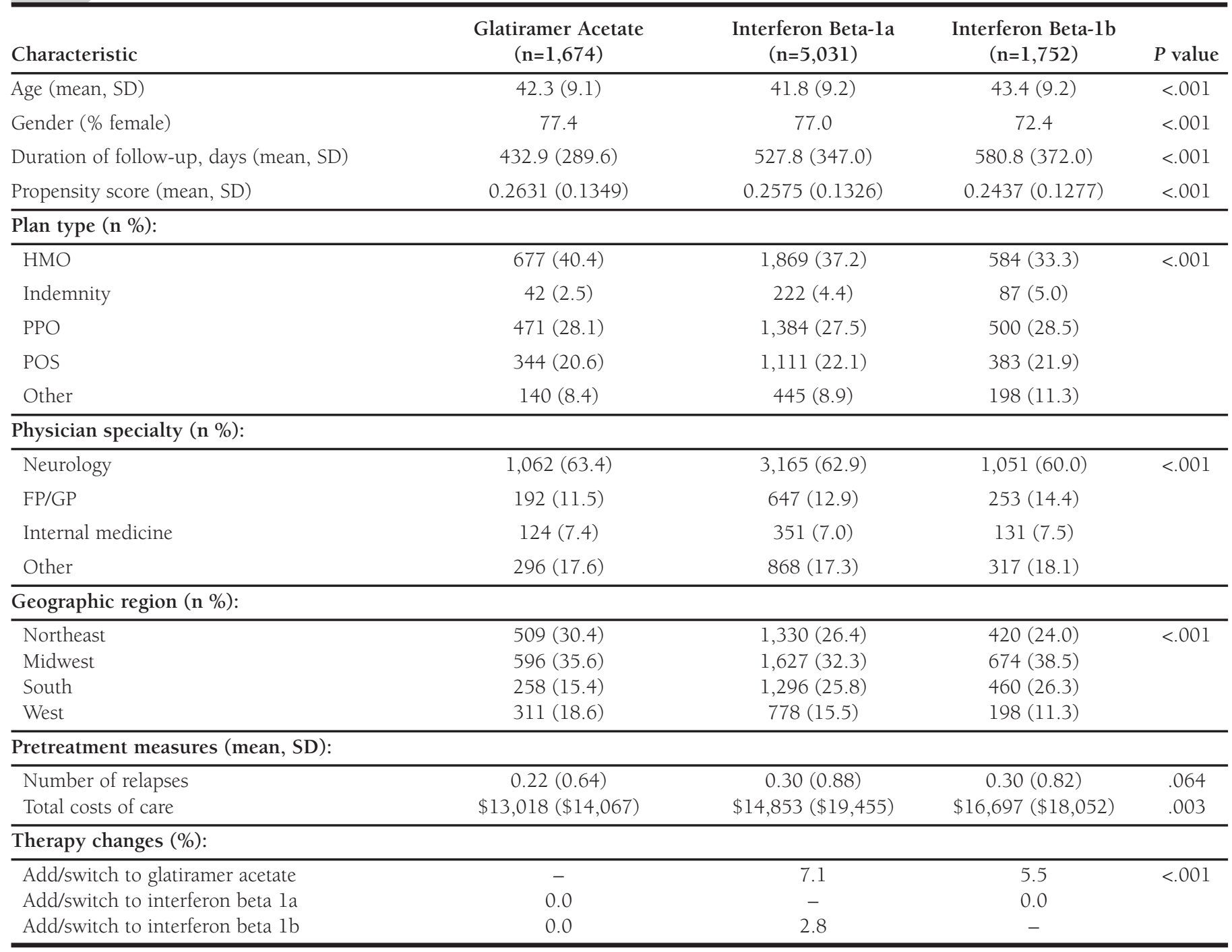

tory therapy were therefore included in these analyses. Additional analyses focused attention on important subgroups, including patients newly starting immunomodulatory therapy as well as those remaining on only a single immunomodulatory medication (i.e., exclusive of switch or add-on therapy). New starts were determined based on the absence of any pharmacy claims for immunomodulatory therapy during a 9-month "pretreatment" period (i.e., prior to the first claim for the medication of interest).

Patients in the 3 treatment groups were first compared with respect to a variety of demographic and clinical characteristics, including age, gender, duration of follow-up, number of relapses and total costs during the pretreatment period (new starts only), type of health plan (e.g., PPO, HMO, fee-for-service), physician specialty, and geographic region.

Estimated propensity scores for use of immunomodulatory therapy also were calculated as a measure of patient severity of ill- ness and disease progression. These scores represent a given patient's probability, or "propensity," of receiving a given treatment option and are calculated by summing coefficient values for a list of potential confounding variables. Use of these scores confers the advantage of having a single estimate available to adjust for confounding (i.e., effects on the findings of interest other than treatment effect) in any multivariate analysis, and have been widely used in clinical and economic research examining causal effects and in comparisons of nonrandomized groups. ${ }^{28,29}$ In this case, the outcome of interest was the probability of use of any of the 3 immunomodulatory medications of interest. Covariates (i.e., potentially confounding variables) were introduced to the model using stepwise logistic regression techniques; those achieving significance at a level of $P<.10$ were retained. In the final model, age, geographic region, a flag for the presence of at least one relapse during the pretreatment and follow-up periods, physi- 
TABLE 2 Relapse Rates and Risk of Relapse by Treatment Group

\begin{tabular}{|c|c|c|c|c|}
\hline Measure & $\begin{array}{l}\text { Glatiramer Acetate } \\
\qquad(\mathrm{n}=1,674)\end{array}$ & $\begin{array}{l}\text { Interferon Beta-1a } \\
\qquad(\mathrm{n}=5,031)\end{array}$ & $\begin{array}{l}\text { Interferon Beta-1b } \\
\quad(\mathrm{n}=1,752)\end{array}$ & $P$ value \\
\hline Cumulative incidence (rate/1,000 person-years) & 291.2 & 314.4 & 241.1 & \\
\hline Time to first relapse, days (mean, SD) & $193.7(160.1)$ & $189.9(163.1)$ & $187.8(157.1)$ & \\
\hline \multirow[t]{3}{*}{$\begin{array}{l}\text { Risk of relapse at one year } \\
\text { (relative to glatiramer acetate)* }\end{array}$} & - & & & \\
\hline & & 1.147 & & .003 \\
\hline & & & 1.512 & .005 \\
\hline
\end{tabular}

cian specialty, and plan type were retained.

Between-group variance in all demographic and clinical measures was assessed using analysis of variance (ANOVA) for continuous variables and an overall chi-square test statistic for proportions.

The cumulative incidence of relapse was presented descriptively as a rate per 1,000 person-years, as was the time to first relapse during follow-up. Hazard rates (i.e., measurement of the increased risk) for the risk of relapse at one year were estimated using a Cox proportional hazards model, controlling for treatment group, propensity score, and censoring (i.e., termination of follow-up at less than one year). The number of relapses per patient also was calculated using a variety of univariate statistics and was compared using ANOVA.

Utilization and costs of MS-related medications and health care services were calculated using overall group means and standard deviations and analyzed using ANOVA techniques. In addition, multivariate analyses of cost were undertaken based on the presence of significant differences in demographic and clinical characteristics between treatment groups. Specifically, an accelerated failure time model, which produces estimates of parametric regression models with censored survival data using the maximum likelihood method, was employed to estimate one-year measures of cost. ${ }^{30}$ Costs were log-transformed prior to analysis to account for data skewness; independent variables included age, sex, and propensity score (inclusion of age both in the propensity score and as a separate covariate allowed for greater precision in generating maximum-likelihood estimates than the propensity score alone).

Relapse rates as well as measures of utilization and cost were generated for prespecified subgroups, including patients newly starting immunomodulatory therapy and those remaining on a single immunomodulatory medication only.

All analyses were conducted using Statistical Analysis Software (SAS), version 8.1 (SAS Institute, Cary, NC).

Results

\section{Patient Characteristics}

Demographic and clinical characteristics of the study sample are presented in Table 1. A total of 8,457 patients were selected for inclusion in these analyses ( $\mathrm{n}=1,674,5,031$, and 1,752 for GA, $1 \mathrm{~A}$, and $1 \mathrm{~B}$, respectively). The mean age for the sample was 42 years; approximately $75 \%$ were female. Both age and gender differed significantly $(P<.001)$ by treatment group. The mean duration of follow-up (approximately 520 days or 17.3 months for the overall sample) also differed significantly $(P<.001)$ by group and was substantially lower among patients receiving GA versus those receiving the other drugs; this phenomenon is likely due to GA's later introduction to the MS marketplace. As a result, unadjusted information on relapse, utilization, and costs is presented on an annualized basis.

Mean propensity scores for use of immunomodulatory therapy were highest for GA patients $(0.2631$ versus 0.2575 and 0.2431 for $1 \mathrm{~A}$ and $1 \mathrm{~B}$, respectively). Significant $(P<.001)$ differences also were noted for plan type, physician specialty, and geographic region. Patients receiving GA were more likely to be members of more managed plans (e.g., HMO, PPO), while those receiving $1 \mathrm{~A}$ and $1 \mathrm{~B}$ were somewhat more likely to be in less managed environments (e.g., indemnity, POS). Nearly two thirds of patients receiving immunomodulatory therapy were managed by a neurologist.

Among patients receiving GA, there were no switches to or polytherapy with either of the beta interferons. However, a total of 498 (9.9\%) and 96 (5.5\%) 1A and 1B patients, respectively, switched or added therapy during follow-up. The majority of these therapy changes involved GA (i.e., either as switch or addon therapy).

Of all identified patients receiving immunomodulatory therapy, a total of 3,161 (37.4\%) were identified as new starts (i.e., based on a 9-month pretreatment period with no use of such medication). Among these patients, the mean $( \pm S D)$ number of relapses during pretreatment did not significantly differ by treatment group $(0.22[ \pm 0.64]$ for $G A$ versus $0.30[ \pm 0.88]$ and $0.30[ \pm 0.82]$ for $1 \mathrm{~A}$ and $1 \mathrm{~B}$, respectively; $P=.064$ ). Mean costs during this period were significantly different, however, and were lowest for GA $(\$ 13,018$ versus $\$ 14,853$ and $\$ 16,697$ for $1 \mathrm{~A}$ and $1 \mathrm{~B}$, respectively; $P=.003$ ). 
Clinical and Economic Impact of Glatiramer Acetate Versus Beta Interferon

Therapy Among Patients with Multiple Sclerosis in a Managed Care Population

TABLE 3 Utilization of MS-related Medications and Health Care Services by Treatment Group

\begin{tabular}{|c|c|c|c|}
\hline & $\begin{array}{l}\text { Glatiramer Acetate } \\
\qquad(\mathrm{n}=1,674)\end{array}$ & $\begin{array}{l}\text { Interferon Beta-la } \\
\quad(\mathrm{n}=5,031)\end{array}$ & $\begin{array}{l}\text { Interferon Beta-1b } \\
\quad(\mathrm{n}=1,752)\end{array}$ \\
\hline Measure (per patient per year) & & Mean (SD) & \\
\hline \multicolumn{4}{|l|}{ Medications (Prescriptions) } \\
\hline Anxiolytics/sedatives/hypnotics & $0.9(3.1)$ & $0.7(2.6)$ & $0.6(2.4)$ \\
\hline Antihistamines and combinations & $0.0(0.1)$ & $0.0(0.2)$ & $0.0(0.1)$ \\
\hline Anticholinergics & $0.0(0.9)$ & $0.0(0.3)$ & $0.0(0.9)$ \\
\hline Anticonvulsants & $0.0(0.2)$ & $0.0(0.2)$ & $0.0(0.4)$ \\
\hline Adrenals and combinations & $1.7(4.9)$ & $1.4(4.0)$ & $1.3(4.0)$ \\
\hline Antidepressants & $2.5(5.1)$ & $2.3(4.9)$ & $2.3(4.7)$ \\
\hline Antivirals & $0.8(2.8)$ & $0.7(2.5)$ & $0.9(3.0)$ \\
\hline Antineoplastic agents & $0.1(0.9)$ & $0.1(0.9)$ & $0.1(0.6)$ \\
\hline Antipsychotics & $0.1(1.0)$ & $0.1(0.8)$ & $0.1(0.7)$ \\
\hline Cerebral stimulants & $0.6(2.3)$ & $0.5(2.3)$ & $0.4(2.0)$ \\
\hline Genitourinary smooth muscle relaxants & $0.4(2.2)$ & $0.5(2.2)$ & $0.6(2.3)$ \\
\hline Hypotensive agents & $0.0(0.4)$ & $0.0(0.3)$ & $0.0(0.2)$ \\
\hline Immunosuppressants & $0.1(1.2)$ & $0.1(1.1)$ & $0.1(1.0)$ \\
\hline Serums & $0.0(0.0)$ & $0.0(0.0)$ & $0.0(0.0)$ \\
\hline Skeletal muscle relaxants & $1.6(4.2)$ & $1.6(4.8)$ & $1.9(4.8)$ \\
\hline Urinary anti-infectives & $0.2(1.2)$ & $0.1(0.9)$ & $0.2(1.1)$ \\
\hline \multicolumn{4}{|l|}{ Other MS-related services } \\
\hline Complete blood counts & $0.7(1.7)$ & $0.9(1.7)$ & $1.0(1.7)$ \\
\hline Platelet counts & $0.1(0.4)$ & $0.2(0.6)$ & $0.2(0.6)$ \\
\hline Liver function tests & $0.6(2.5)$ & $1.0(2.5)$ & $1.0(2.5)$ \\
\hline Emergency-room visits & $0.1(0.5)$ & $0.1(0.6)$ & $0.1(0.4)$ \\
\hline Physician office visits & $3.1(3.6)$ & $3.2(4.5)$ & $2.7(4.3)$ \\
\hline Home health visits & $0.1(1.3)$ & $0.0(0.3)$ & $0.0(0.1)$ \\
\hline Imaging visits & $0.3(0.8)$ & $0.3(0.8)$ & $0.3(0.7)$ \\
\hline Other hospital outpatient visits & $7.8(21.1)$ & $9.6(26.5)$ & $7.0(14.1)$ \\
\hline Hospitalizations & $1.3(8.7)$ & $1.0(4.9)$ & $1.1(4.8)$ \\
\hline
\end{tabular}

\section{Relapse Rates}

Information on relapse rates is presented in Table 2. The cumulative incidence of all relapses during follow-up was 291.2, 314.4 , and 241.1 per 1,000 person-years for GA, 1A, and 1B respectively. Time to first relapse was somewhat longer among patients receiving GA (193.7 $[ \pm 160.1]$ days versus 189.9 $[ \pm 163.1]$ and $187.8[ \pm 157.1]$ for $1 \mathrm{~A}$ and $1 \mathrm{~B}$, respectively); correspondingly, the adjusted one-year risk of relapse was significantly $(P<.005)$ higher for the beta interferons relative to GA $(\mathrm{HR}=1.147$ and 1.512 for $1 \mathrm{~A}$ and $1 \mathrm{~B}$, respectively).

\section{MS-related Resource Utilization}

Utilization of MS-related medications and health care services is presented in Table 3. Other than study therapy, most other medications were used infrequently. The most commonly used medications were antidepressants (mean number of prescriptions per year: 2.3 to 2.5), skeletal muscle relaxants (1.6 to 1.9), and adrenals and combinations (1.3 to 1.7 ). Surprisingly, while periodic laboratory monitoring is recommended for the beta interferons but not for GA, the use of selected tests was infrequent among all groups and did not materially differ between 
Clinical and Economic Impact of Glatiramer Acetate Versus Beta Interferon

Therapy Among Patients With Multiple Sclerosis in a Managed Care Population

\begin{tabular}{lcccc}
\hline TABLE 4 Costs of MS-related Medications and Health Care Services by Treatment Group & \\
\hline Measure & $\begin{array}{c}\text { Glatiramer Acetate } \\
(\mathbf{n}=\mathbf{1}, 674)\end{array}$ & $\begin{array}{c}\text { Interferon Beta-1a } \\
(\mathbf{n}=5,031)\end{array}$ & $\begin{array}{c}\text { Interferon Beta-1b } \\
(\mathbf{n}=1,752)\end{array}$ & $\boldsymbol{P}$ value \\
\hline Medications & $\$ 6,740(\$ 5,301)$ & $\$ 7,547(\$ 5,856)$ & $\$ 7,648(\$ 5,968)$ & $<.001$ \\
\hline Study therapy & $\$ 516(\$ 420)$ & $\$ 445(\$ 365)$ & $\$ 435(\$ 292)$ & .764 \\
\hline Other MS-related medications & $\$ 7,256(\$ 5,727)$ & $\$ 7,992(\$ 6,219)$ & $\$ 8,083(\$ 6,260)$ & $<.001$ \\
\hline TOTAL medications & $\$ 1,291(\$ 2,998)$ & $\$ 1,202(\$ 3,171)$ & $\$ 1,083(\$ 2,603)$ & .459 \\
\hline Outpatient care & $\$ 975(\$ 5,575)$ & $\$ 763(\$ 4,029)$ & $\$ 1,019(\$ 4,992)$ & .168 \\
\hline Inpatient care & $\$ 9,522(\$ 9,706)$ & $\$ 9,957(\$ 9,083)$ & $\$ 10,185(\$ 9,526)$ & .004 \\
\hline TOTAL MS-related costs & & & & \\
\hline Note: All cost measures are annualized. & & & &
\end{tabular}

them. Utilization of other services also is presented in Table 3. Use of physician office and other hospital outpatient services was highest among $1 \mathrm{~A}$ patients, while use of inpatient services (i.e., for treatment of relapse and other MS-related services) was slightly higher among GA patients.

\section{MS-related Costs}

Annualized costs of medications and health care services are presented in Table 4. Mean $( \pm S D)$ costs of all MS-related medications were lowest for $\mathrm{GA}(\$ 7,256[ \pm \$ 5,727])$, followed by $1 \mathrm{~A}$ $(\$ 7,992[ \pm \$ 6,219)$ and $1 \mathrm{~B}(\$ 8,083[ \pm \$ 6,260])$; these differences were statistically significant $(P<.001)$. GA's lower costs relative to the beta interferons were manifested almost entirely in lower acquisition costs for study therapy. Costs of outpatient services were similar for GA and 1A and slightly lower for 1B. Costs for inpatient care were lowest for $1 \mathrm{~A}$ relative to the other 3 groups; neither outpatient nor inpatient costs differed statistically. Total costs of care averaged $\$ 9,522( \pm \$ 9,706), \$ 9,957$ $( \pm \$ 9,083)$, and $\$ 10,185( \pm \$ 9,526)$ for $G A, 1 A$, and $1 B$, respectively $(P=.004)$, which again primarily reflects differences in immunomodulatory drug costs. Findings persisted in multivariate analyses of cost; differences were mitigated somewhat between GA and 1A, while differences between GA and 1B were more marked ( $\$ 10,879, \$ 10,968$, and $\$ 11,619$ for GA, $1 \mathrm{~A}$, and 1B, respectively).

\section{Additional Analyses}

Among patients newly starting immunomodulatory therapy, the incidence of all relapses during follow-up was $17 \%$ to $22 \%$ higher with the beta interferons relative to GA (data not shown); while the one-year risk of relapse did not differ between $1 \mathrm{~A}$ and GA, the risk among $1 \mathrm{~B}$ patients was nearly double that of GA $(\mathrm{HR}=1.856, P=.020)$. Findings were similar among patients who did not switch or add on therapy (HR=1.389 for 1B relative to $G A, P=.029$ ).

Not surprisingly, in the cohort of patients newly starting immunomodulatory therapy, utilization of health care services was somewhat lower than for the entire cohort, particularly with respect to inpatient care; utilization of MS-related medications was similar, however (data not shown). On an annualized basis, total costs for GA patients remained lower than for $1 \mathrm{~A}$ and $1 \mathrm{~B}$ patients $(\$ 9,646[ \pm \$ 10,209]$ versus $\$ 9,979[+\$ 10,815]$ and $\$ 10,553[+\$ 12,140]$, respectively); on an adjusted basis, costs for GA and 1A were similar $(\$ 11,310$ and $\$ 11,192$, respectively), and reduced relative to $1 \mathrm{~B}(\$ 12,347)$.

\section{Discussion}

To examine the impact of use of GA therapy on relapse rates as well as MS-related resource utilization and costs relative to that among patients receiving beta interferon $1 \mathrm{~A}$ or $1 \mathrm{~B}$, we undertook a retrospective analysis of medical and pharmacy claims data among a cohort of MS patients receiving these medications. Data on the incidence of relapses was examined for these patients, as was information on the utilization and costs of MSrelated medications, outpatient services, and inpatient care.

The results of this study indicate that use of GA therapy in patients with MS results in a lower rate of relapse relative to those receiving either beta interferon therapies. In addition, therapy with GA appeared to be more "durable" than that of the beta interferons-patients receiving the former did not switch or add on immunomodulatory therapy, while nearly $10 \%$ of those receiving beta interferon therapy did experience a therapy change. Finally, total costs of MS-related care were reduced by $\$ 400$ to $\$ 700$ among GA patients relative to the beta interferons; findings persisted in multivariate analyses controlling for age, sex, and propensity scores for immunomodulatory therapy.

A number of previous studies have evaluated the cost-effectiveness of immunomodulatory therapy in multiple sclerosis. ${ }^{31-37}$ Without exception, all of the existing studies have compared the costs and effects of beta interferon or GA therapy to those among patients receiving therapy for symptomatic relief only. To the best of our knowledge, this is the first such study to com- 


\section{Clinical and Economic Impact of Glatiramer Acetate Versus Beta Interferon Therapy Among Patients With Multiple Sclerosis in a Managed Care Population}

pare clinical and economic effects among these medications specifically. Most economic studies that have been performed to date have concluded that use of immunomodulatory therapy results in a substantial increase in costs and only modest clinical effects. ${ }^{32-36}$ These findings are at odds with current clinical opinion, however, which suggests that immunomodulatory agents should receive widespread use among patients with MS and should be used early in the course of the disease. ${ }^{38}$

\section{Limitations}

We note some limitations of our analysis. First, our comparison was limited to patients receiving immunomodulatory therapy, as noted above. In reality, many MS patients are still treated with only medications for the symptoms; in fact, nearly $75 \%$ of the patients with MS diagnoses in our database did not receive immunomodulatory medications. Examination of the economic and clinical impact of immunomodulatory medications as a group in an MS population requires comparison to a comparable population that did not receive these medications. Such an analysis is likely to be problematic in a retrospective database, however, as patients not receiving immunomodulatory therapy are historically likely to be at an earlier stage of disease progression and therefore less severely ill.

In addition, as with all quasi-experimental research based on retrospective data, we cannot rule out the possibility that our findings may have been influenced by differences in disease severity, duration of illness, and rate of disease progression between the 3 treatment groups that were the focus of our analysis. While it is true that these important factors are not detectable in any detailed way in claims data, the use of a propensity score in this circumstance does provide a method to control for differences in patient, physician, or health plan characteristics between patient groups. Indeed, our findings were nearly identical among the subgroup of patients newly starting immunomodulatory therapy (who would be expected to be more comparable in terms of disease progression and/or severity) as well as those remaining on a single agent during followup. Perhaps most importantly, the direction of these findings was unchanged in multivariate analyses controlling for propensity for immunomodulatory therapy and other covariates that were detectable in this particular data source.

In addition, our measure of relapse was limited to utilization proxies only; this definition was likely insensitive or conservative since overall annualized relapse rates $(0.24$ to 0.31 per patient) are lower than those reported in clinical studies. ${ }^{4-14,39}$ However, this effect was equally distributed across treatment groups and therefore likely affects only the magnitude (and not the direction) of our findings. While GA appeared to confer some benefit with regard to relapse as measured in our study, it should be noted that differences in risk and time to event were moderate (albeit statistically significant); perceptions as to the clinical significance of these differences will vary.

Finally, we could not measure the impact of these medica- tions on important intermediate and qualitative outcomes such as lesion detection on magnetic resonance imaging, disability status, or function. Measurement of clear differences in effectiveness will only come through the conduct of randomized, head-to-head clinical trials of these agents, where levels of disease severity are comparable at the time of enrollment.

\section{Conclusion}

Our findings suggest that use of glatiramer acetate may reduce rates of relapse as well as levels of MS-related utilization and costs relative to the beta interferons; further study is required, however, to document these benefits in a definitive manner. Our results are likely to be of interest to health care payers and providers as well as patients with multiple sclerosis who may be candidates for immunomodulatory therapy.

\section{DISCLOSURES}

Funding for this research was provided by Teva Neuroscience, Inc., U.S., a subsidiary of Teva Pharmaceutical Industries, Ltd., manufacturer of glatiramer acetate. Author Merri Kay Oleen-Burkey is employed by Teva Neuroscience, Inc. Authors Daniel A. Ollendorf and Evguenia Jilinskaia are employed by PharMetrics, Inc., a health care data and research company that has consulting contracts with Teva Neuroscience. Funding was obtained by Ollendorf. Ollendorf served as principal author of the study. Study concept and design and drafting of the manuscript were contributed primarily by Ollendorf and Oleen-Burkey. Critical revision of the manuscript was the work of Jilinskaia and Oleen-Burkey. Analysis and interpretation of data were contributed primarily by Ollendorf and Jilinskaia. Statistical expertise was contributed by Jilinskaia. Administrative, technical, and/or material support was provided by PharMetrics.

\section{REFERENCES}

1. Weinshenker BG. Epidemiology of multiple sclerosis. Neurol Clin. 1996;14:291-308.

2. Weinshenker BG. The natural history of multiple sclerosis. Neurol Clin. 1995;13:119-46.

3. Pope GC, Urato CJ, Kulas ED, et al. Prevalence, expenditures, utilization, and payment for persons with MS in insured populations. Neurology. 2002;58:37-43.

4. Oentrich W, Dose T, Friedmann D, et al. Interferon beta-1b therapy in patients with relapsing-remitting multiple sclerosis: findings of a prospective, multicenter study of disease progression. Nervenarzt. 2001;72:286-92.

5. Johnson KP, Brooks BR, Cohen JA, et al. and the Copolymer 1 Multiple Sclerosis Study Group. Copolymer 1 reduces relapse rate and improves disability in relapsing-remitting multiple sclerosis. Neurology. 1995;45:1268-76

6. Johnson KP, Brooks BR, Cohen JA, et al. and the Copolymer 1 Multiple Sclerosis Study Group. Extended use of glatiramer acetate (Copaxone) is well tolerated and maintains its clinical effect on multiple sclerosis relapse rate and degree of disability. Neurology. 1998;50:701-08.

7. Johnson KP, Brooks BR, Ford CC, et al. and the Copolymer 1 Multiple Sclerosis Study Group. Sustained clinical benefits of glatiramer acetate in relapsing multiple sclerosis patients observed for 6 years. Multiple Sclerosis. 2000;6:255-66

8. The IFNB Multiple Sclerosis Study Group. Interferon beta-1b is effective in relapsing-remitting multiples sclerosis: clinical results of a multicenter, randomized double-blind, placebo-controlled trial. Neurology. 1993;43:655-61.

9. The IFNB Multiple Sclerosis Group and the University of British Columbia MS/MRI Analysis Center. Interferon beta-1b in the treatment of multiple sclerosis: final outcome of the randomized controlled trial. Neurology. 
1995;45:1277-85.

10. Jacobs LD, Cookfair DL, Rudick RA, et al. and the Multiple Sclerosis Collaborative Research Group (MSCRG). Intramuscular interferon beta-la for disease progression in relapsing multiple sclerosis. Ann Neurol. 1996;39:285-94.

11. Rudick RA, Goodkin DE, Jacobs LD, et al. and the Multiple Sclerosis Collaborative Research Group (MSCRG). Impact of interferon beta-la on neurologic disability in relapsing multiple sclerosis. Neurology. 1997;49:358-63.

12. La Mantia L, Milanese C, D’Amico R. Meta-analysis of copolymer 1 in multiple sclerosis. Eur Neurol. 2000;43:189-93.

13. Ge Y, Grossman RI, Udupa JK, et al. Glatiramer acetate (Copaxone) treatment in relapsing-remitting MS: quantitative MR assessment. Neurology. 2000;54:813-17.

14. Liu C, Blumhardt LD. Benefits of glatiramer acetate on disability in relapsing-remitting multiple sclerosis: an analysis by area under disability/time curves. The Copolymer 1 Multiple Sclerosis Study Group. J Neurol Sci. 2000;181:33-37.

15. Liu Z, Pelfrey CM, Cotleur A, et al. Immunomodulatory effects of interferon beta-la in multiple sclerosis. J Neuroimmunol. 2001;112:153-62.

16. Kozovska ME, Hong J, Zang YC, et al. Interferon beta induces T-helper 2 immune deviation in MS. Neurology. 1999;53:1692-97.

17. Yong VW, Chabot S, Stuve O, et al. Interferon beta in the treatment of multiple sclerosis: mechanisms of action. Neurology. 1998;51:682-89.

18. Weinstock-Guttman B, Ransohoff RM, Kinkel RP, et al. The interferons: biological effects, mechanisms of action, and use in multiple sclerosis. Ann Neurol. 1996;37:7-15.

19. Neuhaus O, Farina C, Wekerle H, et al. Mechanisms of action of glatiramer acetate in multiple sclerosis. Neurology. 2001;56:702-08.

20. Chen M, Gran B, Costello K, et al. Glatiramer acetate induces a Th2biased response and crossreactivity with myelin basic protein in patients with MS. Multiple Sclerosis. 2001;7:209-19.

21. Gran B, Tranquill LR, Chen M, et al. Mechanisms of immunomodulation by glatiramer acetate. Neurology. 2000;55:1704-14.

22. Duda PW, Krieger JI, Schmied MC, et al. Human and murine CD4 T cell reactivity to a complex antigen: recognition of the synthetic random polypeptide glatiramer acetate. J Immunol. 2000;165:7300-07.

23. Aharoni R, Teitelbaum D, Leitner O, et al. Specific Th2 cells accumulate in the central nervous system of mice protected against experimental autoimmune encephalomyelitis by copolymer 1. Proc Natl Acad Sci USA. 2000;97:11472-11477.

24. Kipnis J, Yoles E, Porat Z, et al. T-cell immunity to copolymer 1 confers neuroprotection on the damaged optic nerve: possible therapy for optic neuropathies. Proc Natl Acad Sci USA. 2000;97:7446-451.

25. Sela M, Teitelbaum D. Glatiramer acetate in the treatment of multiple sclerosis. Expert Opinion Pharmacother. 2001;2:1149-65.
26. Wingerchuk DM, Noseworthy JH, Weinshenker BG. Clinical outcome measures and rating scales in multiple sclerosis trials. Mayo Clin Proc. 1997;72:107079.

27. Schwartz CE, Vollmer T, Lee H. Reliability and validity of two self-report measures of impairment and disability for MS. North American Research Consortium on Multiple Sclerosis Outcomes Study Group. Neurology. 1999;52:63-70

28. Rubin DB. Estimating causal effects from large data sets using propensity scores. Ann Intern Med.1997;127:757-63.

29. D'Agostino RB Jr. Propensity score methods for bias reduction in the comparison of a treatment to a non-randomized control group. Statist Med. 1998; 17:2265-81

30. Lin DY. Proportional means regression for censored medical costs. Biometrics. 2000;56:775-78

31. Bose U, Ladkani D, Burrell A, et al. Cost-effectiveness analysis of glatiramer acetate in the treatment of relapsing-remitting multiple sclerosis. J Med Econ. 2001;4:207-19.

32. Nuijten MJ, Hutton J. Cost-effectiveness analysis of interferon beta in multiple sclerosis: a Markov process analysis. Value Health. 2002;5:44-54.

33. Parkin D, Jacoby A, McNamee P, et al. Treatment of multiple sclerosis with interferon beta: An appraisal of cost-effectiveness and quality of life. J Neurol Neurosurg Psychiatr. 2000;68:144-49.

34. Forbes RB, Lees A, Waugh N, Swingler RJ. Population-based cost utility study of interferon beta- $1 \mathrm{~b}$ in secondary progressive multiple sclerosis. BMJ. 1999;319:1529-33.

35. Brown MG, Murray TJ, Sketris IS, et al. Cost-effectiveness of interferon beta-lb in slowing multiple sclerosis disability progression-first estimates. Int J Technol Assess Health Care. 2000;16:751-67.

36. Clegg A, Bryant J. Immunomodulatory drugs for multiple sclerosis: a systematic review of clinical and cost effectiveness. Expert Opinion Pharmacother. 2001;2:623-39.

37. Kobelt G, Jonsson L, Henriksson F, et al. Cost-utility analysis of interferon beta-1b in secondary progressive multiple sclerosis. Int J Technol Assess Health Care. 2000;16:768-80.

38. van den Noort S, Eidelman B, Rammohan K, et al. National Multiple Sclerosis Society (NMSS): Disease Management Consensus Statement. New York: NMSS; 1998.

39. Khan OA, Tselis AC, Kamholz JA, et al. A prospective, open-label treatment trial to compare the effect of IFNbeta-la (Avonex), IFNbeta-1b (Betaseron), and glatiramer acetate (Copaxone) on the relapse rate in relapsing-remitting multiple sclerosis: Results after 18 months of therapy. Multiple Sclerosis. 2001;7:349-53. 